EESTI NSV TEADUSTE AKADEEMIA TOIMETISED. 31. KOIDE FOOSIKA * MATEMAATIKA. 1932, NR. 4

ИЗВЕСТИЯ АКАДЕМИИ НАУК ЭСТОНСКОИ ССР. ТОМ ЗІ ФИЗИКА * МАТЕМАТИКА. 1982, № 4

удК 539.12

R.-K. LOIDE

\title{
ON THE DEGREE OF THE MINIMAL EQUATION FOR FIRST-ORDER WAVE EQUATIONS
}

\author{
R. - K. LOIDE. MINIMAALSE VORRANDI ASTE ESIMEST JARKU LAINEVORRANDITELE \\ P.К. ЛОНДЕ. О СТЕПЕНИ МИННМАЛЬНОГО УРАВНЕНИЯ ДЛЯ ВОЛНОВЫХ УРАВНЕНИН \\ ПЕРВОГО ПОРЯДКА
}

(Presented by A. Humal)

In this note we deal with the first-order single-mass wave equations

$$
\left(p_{\mu} \beta^{\mu}-m\right) \psi=0 .
$$

As it was shown in $\left[{ }^{1}\right]$, the minimal equation of $\beta^{0}$ matrix must have the form

$$
\left(\beta^{0}\right)^{L}\left(\left(\beta^{0}\right)^{2}-I\right)=0 .
$$

Proceeding from the quite general assumptions, Umezawa and Visconti proved that $L=2 s_{m}-1$, where $s_{m}$ is the maximum spin in $\psi$ representation $\left[{ }^{2}\right]$. Although the latter relation is valid in the case of many equations, it is not universal. In $\left[{ }^{3}\right]$ it was demonstrated that it is possible to get equations, where $L>2 s_{m}-1$. The Umezawa-Visconti condition was also analysed in $\left[{ }^{4}\right]$, where it was proved that $2 s-1 \leqslant L \leqslant$ $\leqslant 2 s_{m}-1$ ( $s$ is the physical spin in (1)). As it was demonstrated in $\left[{ }^{5}\right]$, there are no general spin-dependent expression for $L$, the degree of the minimal equation (2) depends on a general matrix structure of $\beta^{0}$.

The problem of the degree of the minimal equation becomes actual due to the consistency problem of high spin wave equations. It turns out that in the presence of external fields equation (1) becomes acausal if $\beta$-matrices contain nilpotent sub-blocks $(L>1)$. Therefore the acausality depends on the general algebraic structure of $\beta$-matrices and on the knowledge of upper and lower bounds on $L$. In some recent papers $\left[{ }^{6-8}\right]$ the restrictions on $L$ were re-examined anew. As we shall show, the result's based only on group-theoretical arguments are not, in general, valid. One must take into consideration also the number of irreducible representations with a given spin $s$ and the existence of free parameters.

Let us examine the general structure of $\beta^{0}$ matrix and clarify the restrictions which one must take into consideration calculating $L$. Suppose $\psi$ is decomposed into direct sum of irreducible representations $i=$ $=\left(k_{i}, l_{i}\right): 1 \oplus 2 \oplus \ldots \oplus r$. Then $\beta^{0}$ is written in the following general form $\left[{ }^{5}\right]-$ 


$$
\beta^{0}=\left|\begin{array}{cccc}
a_{11} t_{11} & a_{12} t_{12} & \ldots & a_{1 r} t_{1 r} \\
a_{21} t_{21} & a_{22} t_{22} & \ldots & a_{2 r} t_{2 r} \\
\cdot & \ddots & & \cdot \\
\cdot & \ddots & & \vdots \\
a_{r 1} t_{r 1} & a_{r 2} t_{r 2} & \ldots & a_{r r} t_{r r}
\end{array}\right|
$$

where $a_{i j}$ are arbitrary free parameters. $t_{i j}$ are matrices which are expressed with the help of spin projection operators $t_{i j}^{s}$

$$
t_{i j}=\sum_{s} \alpha_{i j}(s) t_{i j}^{s}
$$

where the summing is over all common spins in $i$ and $j$. Only these $t_{i j}$ are nonzero which correspond to linked representations $i$ and $j$. Coefficients $\alpha_{i j}(s)$ are uniquely determined by $i$ and $j\left[{ }^{5}\right]$.

Ii is useful to decompose $\beta^{0}$ into the sum $\left[{ }^{5,9}\right]$

$$
\beta^{0}=\beta^{s_{1}-1}-\beta^{s_{2}}+\ldots+\beta^{s_{k}},
$$

where $\beta^{s}$ includes spin projection operators $t_{i j}^{s}$ of a given spin $s$. The investigation of minimal equation (2) reduces to the investigation of minimal equations of $\beta^{s}$. As it is shown in $\left[{ }^{5,9}\right]$, the minimal equations of $\beta^{8}$ are:

1) equation (1) describes particles with spin $s_{i}$

$$
\left(\beta^{s_{t}}\right)^{a_{t}}\left(\left(\beta^{s_{t}}\right)^{2}-I\right)=0,
$$

where $a_{i} \geqslant 1$ ( $a_{i}=0$ only in the case of Dirac bispinor);

2) equation (1) do not describe particles with spin $s_{j}$

$$
\left(\beta^{s_{t}}\right)^{b_{t}}=0 \text {, }
$$

where $b_{j} \geqslant 2$. The degree $L$ has a general upper bound

$$
L \leqslant \max \left\{a_{i}, b_{i}\right\} \text {. }
$$

The reason why some of the $\beta^{s}$ must be nilpotent and satisfy (7) or contain nilpotent sub-blocks (if $a_{i}>1$ ) lies in the fact that in general the decomposition (5) contains more spins than one wants to describe by equation (1).

In $\left.{ }^{5}\right]$ we have shown that minimal equations of $\beta^{s}$ are limited by the number of irreducible representations $\left(k_{i}, l_{i}\right)$ which carried spin $s$. When there are $n$ irreducible representations which carried spin $s$, the investigation of $\beta^{s}$ reduces to the investigation of some $n \times n$ matrix formed from $a_{i j} \alpha_{i j}(s)$. The upper and lower bounds for $a_{i}$ and $b_{j}$ depend on the dimensionality $n$ and on the choice of free parameters $a_{i j}$, and therefore have not uniquely determined. The maximal upper bound is determined by the maximal dimensionality $n_{m}$ of reduced $\beta^{s}$ matrices, and $L$ cannot exceed $n_{m}$. Analogical results are also given in $\left[{ }^{6}\right]$, using similar arguments to those in $\left[{ }^{5}\right]$.

As we have mentioned, the minimal equation of $\beta^{0}$ depends also on the existence of free parameters $a_{i j}$. In general the number of independent parameters is restricted, it depends on a given representation, linkage schema and additional restrictions, as the parity conservation and derivability from lagrangian. As it is shown in $\left[{ }^{10}\right]$, the number of free parameters restricts the structure of possible equations with a given representation. The representation $(1,1 / 2) \oplus(0,1 / 2) \oplus(1 / 2,0) \oplus(1 / 2,1)$, for example, admits three types of single-mass equations with $L=2,1$ and 0 , respectively $\left[5,9,1^{0}\right]$. Here we have no spin-dependent expression for $L$. 
Also, the lower bound proposed in $\left[{ }^{7}\right]$ does not take place. From $\left[{ }^{7}\right]$ one must have $L \geqslant 2 \cdot 3 / 2-1=2$.

In $\left[{ }^{8}\right]$ it is supposed that one has a general lower bound $L>2 s-1$, where $s$ is a physical spin in (1). The representation $(s, 0) \oplus(s \pm 1 / 2,1 / 2)$ and parity-doubled representation gives us the example where $L=1$ independently on spin $s$ ( $L=0$ only for Dirac bispinor), so the above condition is not in general valid.

The restrictions on $L$ given in $[3,4,7,8]$ are derived using similar general arguments to those in the original work of Umezawa and Visconti $\left[{ }^{2}\right]$. These proofs based on the existence of Klein-Gordon divisor and its general group-theoretical transformation properties. Since the existence of independent parameters is not taken into consideration, the results are not, in general, valid. Also the supposition that Klein-Gordon divisor transforms like $\psi \otimes \psi$. or like symmetrical tensor contained in $\psi \otimes \psi$, is not correct. Klein-Gordon divisor $d(p)$ is a polynomial in $\left(p_{\mu} \beta^{\mu}\right)\left[^{5,7}\right]$, and in Lorentz transformations $p \rightarrow p^{\prime}$ we get $d(p) \rightarrow d\left(p^{\prime}\right)$. Also, the transformation properties of $\beta^{\mu}, \beta^{\mu} \beta^{v}, \ldots$ are not correctly interpreted. $S^{-1}(\Lambda) \beta^{\mu} S(\Lambda)=\Lambda_{v}^{\mu} \beta^{v}$ is an invariance condition of equation (1) and means nothing more than the $\psi$ representation is composed of linked irreducible representations, i. e. that $S \otimes(1 / 2,1 / 2) \supset S$.

\section{REFERENCES}

1. Harish-Chandra, Phys. Rev., 71. 793-805 (1947).

2. Umezawa. H., Visconti, A., Nucl. Phys., 1, 348 (1956).

3. Glass, A. S., Communs Math. Phys., 23, 176-184 (1971).

4. Chandrasekharan, P. S., Menon, N. B., S a nthanam, T. S., Progr. Theor. Phys.. 47, № 2, 671-677 (1972).

5. Lo ide, K., Lo ide, R.-K., Preprint F-6, Tartu, 1977.

6. Mathews, P. M., Seetharaman, M., Takahashi, Y., J. Phys. A: Math. Gen., 13, № 9, 2863-2872 (1980).

7. Mathews, P. M., Govindarajan, T. R., Preprint MUTP-81/10, Madras, 1981.

8. Cox, W., J. Phys. A: Math. Gen., 14, № 9, 2459-2465 (1981).

9. Л ойде Р.-К., Изв. АН ЭССР, Физ. Матем.. 23, № 3, 203-209 (1974).

10. Kõ iv, M., Loide, R. - K., S a a r, R., ENSV TA Toim., Füüs. Matem., 31, № 3, $300-303$ (1982).

Tallinn Polytechnic Institute

Received

Febr. 17, 1982 